\title{
Estrategias en la resolución de problemas algebraicos en un contexto intercultural en el nivel superior
}

\author{
Strategies in solving algebraic problems in an intercultural context in \\ Higher Education
}

\author{
Javier García-García* \\ ORCID iD 0000-0003-4487-5303
}

\begin{abstract}
Resumen
El presente escrito tiene por objetivo caracterizar las estrategias que utilizan los estudiantes universitarios en un contexto intercultural cuando resuelven problemas algebraicos, previo al tratamiento formal del tema de ecuaciones lineales en este nivel educativo. La investigación adopta un marco conceptual; se definen los constructos estrategias (reflexivas e irreflexivas), problemas y problemas algebraicos. Es un estudio de cinco casos. Para la colecta de datos se utilizaron cuestionarios escritos que plantearon cinco problemas algebraicos y entrevistas audio grabadas. Los resultados indican que los estudiantes utilizan siete estrategias reflexivas (por ejemplo, se apoya de hechos conocidos y genera relaciones, realiza un trabajo hacia atrás, entre otras) y una estrategia irreflexiva, algunas de las cuales han sido identificadas en otras investigaciones. Los resultados invitan a incorporar algunas de las estrategias personales de los estudiantes durante la enseñanza formal en el aula de clases de Matemáticas, como una forma de practicar la interculturalidad y promover la participación de los estudiantes.
\end{abstract}

Palabras clave: Estrategias. Resolución de problemas algebraicos. Interculturalidad. Estudio de casos. Nivel Superior.

\begin{abstract}
This paper aims to characterize the strategies that university students use in an intercultural context when solving algebraic problems, previous to the formal instruction of the linear equations topic in this educational level. The research adopts a conceptual framework; defining strategies (reflexive and unreflective), problems and algebraic problems. This is a five-case study. We used written questionnaires which raised five algebraic problems and recorded audio interviews for collecting data. The results indicate that students use seven reflexive strategies (for example, they support on known facts and generate relationships, make backward work, among others) and an unreflective strategy, some of which have been identified in other researches. The results obtained invite to incorporate some of the students' personal strategies during formal instruction in the Mathematics classroom as a way of practicing the interculturality and encouraging students' participation in the classroom.
\end{abstract}

Keywords: Strategies. Solving algebraic problems. Interculturality. Case studies. Higher education.

\section{Introducción}

\footnotetext{
* Doctor en Ciencias en el Área de Matemática Educativa por la Universidad Autónoma de Guerrero (UAGro). Profesor-investigador en el Centro de Investigación en Matemática Educativa (CIMATE-UAGro), Chilpancingo, Guerrero, México. Dirección postal: Avenida Lázaro Cárdenas S/N, Col. La Haciendita, Chilpancingo de los Bravo, Guerrero, México, C.P: 39087. E-mail: jagarcia@uagro.mx.
} 
México es un país pluricultural que cuenta con aproximadamente 62 grupos étnicos con costumbres e idioma propio (con sus respectivas variantes) (GARCÍA-GARCÍA; RODRÍGUEZ; NAVARRO, 2015). Esto ha permitido que la retórica oficial reconozca y promueva la interculturalidad en el ámbito educativo.

En niveles básicos (primaria y secundaria) se declara, explícitamente, en los planes y programas de estudio su importancia; mientras que para bachillerato y nivel superior se han creado escuelas interculturales que, prioritariamente atienden a la población étnica que es rechazada de escuelas convencionales. En el nivel superior, por ejemplo, las Universidades Interculturales tienen como misión: promover la revitalización, desarrollo y, consolidación de lenguas y culturas originarias.

En este escrito, se asume que la población estudiantil hablante de una lengua originaria requiere ser estudiada desde diferentes enfoques para comprender su proceso de aprendizaje y los factores que inciden en su rendimiento, tal como lo sugieren García (2013), García-García (2014a, 2014b) y, García-García, Navarro y Rodríguez (2014). Esto porque la enseñanza y el aprendizaje de las Matemáticas es más compleja en un salón pluricultural, sin importar el nivel educativo. Este es el caso de la Universidad Intercultural del Estado de Guerrero (UIEG), donde acuden estudiantes hablantes de al menos cuatro lenguas originarias.

Por otra parte, se asume que en la resolución de problemas se debe trascender más allá de algoritmos, y es necesario fomentar en el aula de clases el uso de estrategias. Esta tarea no es trivial, porque al parecer, los alumnos se han acostumbrado a resolver problemas de manera automática, sin previo análisis. Por ejemplo, a un grupo de estudiantes de la UIEG se les planteo este problema: un pastor tiene 125 ovejas y 5 perros. ¿Qué edad tiene el pastor? (tomado de RIZO; CAMPISTROUS, 1999). Aun cuando el problema es irresoluble con los datos dados, la mayoría (cerca del 97\%) realiza un trabajo operatorio con los datos, el más común una división de 125 entre 5, cuyo resultado dan como respuesta. Resultados como estos orientan a seguir investigando en esta área, para identificar por qué el estudiante procede como lo hace en la resolución de problemas.

La literatura especializada indica que la resolución de problemas ha sido explorada en numerosas poblaciones estudiantiles de distintos niveles educativos, baste como ejemplo señalar a Fernández (2006), Bermejo y Díaz (2007), Villalobos (2008), y Molina y Ambrose (2010).

Asimismo, existen estudios que exploran las estrategias utilizadas al resolver diversas tareas matemáticas (RIZO; CAMPISTROUS, 1999; ELIA; HEUVEL-PANHUIZEN; KOLOVOU, 2009; CHE; WIEGERT; THRELKELD, 2012; JIANG; CHUA, 2010; GARCÍA, 
2013; GARCÍA-GARCÍA; NAVARRO; RODRÍGUEZ, 2014; GARCÍA-GARCÍA, 2014a, 2014b; GARCÍA-GARCÍA; RODRÍGUEZ; NAVARRO， 2015; OBERSTEINER; BERNHARD; REISS, 2015). De estos estudios, sólo García-García (2014b) explora el razonamiento que siguen los estudiantes indígenas de nivel superior al resolver problemas geométricos. Por lo tanto, en este nivel educativo se requiere hacer más investigaciones para tener un panorama amplio respecto de su rendimiento al resolver tareas matemáticas.

Es en esta ruta en la que se plantea el presente trabajo. Se parte de que es importante ubicar las estrategias personales de los alumnos para incorporarlas en el aula de clases como un medio para significar los conceptos matemáticos que se aborden, además de que podrían ser aprovechadas en la enseñanza tal como lo sugiere García-García, Navarro y Rodríguez (2014). Por lo tanto, se planteó como pregunta de investigación ¿cuáles son las estrategias que utilizan los estudiantes universitarios en un contexto intercultural para resolver problemas algebraicos, previo al tratamiento formal del tema de ecuaciones lineales en este nivel educativo?

\section{Marco Conceptual}

Los constructos teóricos sobre los que se fundamenta en este estudio son: estrategia, problemas y problemas algebraicos. Estos se clarifican enseguida.

\subsection{El constructo estrategia}

El término estrategia en el campo educativo fue retomado por los años setenta, creyéndose que podría contribuir a resolver el problema de aprender a aprender (DÍAZBARRIGA; HERNÁNDEZ, 2010). Desde entonces, ha jugado un rol importante en la práctica docente, tanto en la enseñanza-aprendizaje como en la evaluación. Es así como en el contexto escolar se puede hablar de estrategias de aprendizaje, de enseñanza, de evaluación y estrategias en la resolución de problemas. Este último es el tipo de estrategia que interesa en este trabajo.

Para efectos de este trabajo, se asume que una estrategia es un conjunto de acciones intencionales desarrolladas por un alumno para resolver cierto problema. Estas están permeadas por los conocimientos de que dispone el estudiante, de su experiencia, de lo afectivo y del contexto social en el que se desenvuelve. Por tanto, podrá llegar o no a la solución del problema; esto dependerá del nivel de análisis que realice de la situación descrita 
en el texto del problema (GARCÍA, 2012, 2013).

Las acciones a las que se refiere García (2012, 2013) son procedimientos intencionales, por ejemplo, apoyarse de un trabajo operatorio identificando previamente los datos y la operación a efectuar, realizar una operación mental o recurrir a explicaciones verbales o gestuales. Las estrategias pueden ser identificadas en las producciones escritas y/o en los argumentos verbales o gestuales que acompañan a la actividad desarrollada por los estudiantes.

Por otra parte, por el análisis que desarrolla un estudiante al resolver problemas se reconoce la existencia de estrategias reflexivas e irreflexivas (RIZO; CAMPISTROUS, 1999). Las reflexivas se presentan cuando los estudiantes desarrollan previamente un proceso de análisis para encontrar la solución. Mientras que las segundas, corresponden a un proceder prácticamente automatizado, sin que pase por un proceso previo de análisis u orientación en el problema (RIZO; CAMPISTROUS, 1999).

Para efectos de este trabajo se resumieron algunas condiciones necesarias para identificar si el estudiante realizó o no un análisis del problema previo a encontrar la solución (Cuadro 1). Estas se presentan enseguida.

\begin{tabular}{|c|c|}
\hline Tipo de estrategia & $\begin{array}{l}\text { ¿Cuándo se cae en su uso? } \\
\text { Emerge cuando el alumno: }\end{array}$ \\
\hline Estrategias reflexivas & $\begin{array}{l}\text { - } \quad \text { Comprende lo que plantea la situación general. } \\
\text { - } \quad \text { Pbica correctamente los datos del problema. } \\
\text { - Sabe explecuadamente el plá a hizo, cómo y por qué. } \\
\text { - Llega a la solución del problema y verifica la validez de su resultado. }\end{array}$ \\
\hline $\begin{array}{l}\text { Estrategias } \\
\text { irreflexivas }\end{array}$ & $\begin{array}{l}\text { - Tiene alguna dificultad para comprender lo que plantea la situación. } \\
\text { - Pbica total o parcialmente los datos que presenta el problema. } \\
\text { solución, pero aun consí realiza operaciones aritméticas. } \\
\text { - Generalmente, desconoce por qué procede como lo hace. } \\
\text { Llega a una solución que puede ser consistente o no desde el punto } \\
\text { de vista matemático. }\end{array}$ \\
\hline
\end{tabular}

Cuadro 1 - Tipología de estrategias y su caracterización Fuente: elaborado por el autor

Además de las características descritas en el Cuadro 1, en el caso de las estrategias reflexivas, los alumnos pueden llegar a una respuesta incorrecta por errores algorítmicos. Mientras que, en las irreflexivas llegar a respuestas correctas, pero la explicación que ofrecen no es consistente desde el punto de vista matemático.

\subsection{El constructo problema}

En el aula de clases, normalmente se dedica un espacio para resolver problemas 
matemáticos. Constructo cuya definición no es la misma para todos los investigadores en Matemática Educativa. Esta dificultad reside en que el término es subjetivo, es decir, lo que para un individuo es un problema, para otro no pasa de ser un mero ejercicio. En ese sentido, Ortiz (2001, p. 58) plantea que una situación es problema "sólo si el sujeto o los sujetos lo visualizan como tal". Al respecto, la literatura plantea múltiples posturas en relación con el constructo problema (ver Cuadro 2).

\begin{tabular}{|c|c|}
\hline Autor & Caracterización \\
\hline $\begin{array}{l}\text { Schoenfeld } \\
(1985)\end{array}$ & Una tarea que resulta difícil para la persona que está tratando de hacerla. \\
\hline $\begin{array}{l}\text { Rizo y } \\
\text { Campistrous } \\
(1999)\end{array}$ & $\begin{array}{l}\text { Toda situación en la que hay un planteamiento inicial y una exigencia que obliga a } \\
\text { transformarla. La vía de solución tiene que ser desconocida y la persona quiere realmente } \\
\text { realizar la transformación. }\end{array}$ \\
\hline $\begin{array}{l}\text { Cabañas } \\
(2000, \text { p. } 8)\end{array}$ & $\begin{array}{l}\text { Una situación o tarea que intenta transformar o resolver conscientemente un individuo; } \\
\text { que de hecho es una contradicción que se le presenta al individuo y éste quiere resolverla; } \\
\text { y que la vía de solución es desconocida para el individuo. }\end{array}$ \\
\hline Santos (2010) & $\begin{array}{l}\text { Es una tarea o situación que reúne los siguientes componentes: } \\
\text { - La existencia de un interés. } \\
\text { - La no existencia de una solución inmediata; } \\
\text { - La presencia de diversos caminos o métodos de solución; y } \\
\text { - La atención por parte de una persona o un grupo de individuos para llevar a cabo } \\
\text { un conjunto de acciones tendentes a resolver esa tarea. }\end{array}$ \\
\hline $\begin{array}{l}\text { Echenique } \\
(2006, \text { p. 20). }\end{array}$ & $\begin{array}{l}\text { Es una situación que un individuo o grupo quiere o necesita resolver y para la cual no } \\
\text { dispone, en principio, de un camino rápido y directo que le lleve a la solución }[\ldots] \text {. }\end{array}$ \\
\hline $\begin{array}{l}\text { García-García; } \\
\text { Rodríguez; } \\
\text { Navarro } \\
(2015)\end{array}$ & $\begin{array}{l}\text { Una tarea o situación que reúne los siguientes componentes: } \\
\text { - existe una demanda o acción a realizar, para la cual existe una persona o grupo de } \\
\text { personas que quieren o necesitan cumplimentarla. La demanda será adecuada al } \\
\text { nivel de formación de la(s) persona(s); } \\
\text { - hay un proceso que hay que poner en juego para cumplir la demanda, pero que en } \\
\text { primera instancia parece desconocido, es decir, se necesita realizar cierto proceso } \\
\text { de análisis para comprender lo que se pregunta y la situación en general; } \\
\text { - la situación puede tener varios, uno o ningún resultado final, lo cual deberá } \\
\text { determinar la persona haciendo uso de alguna estrategia. }\end{array}$ \\
\hline
\end{tabular}
Cuadro 2 - Definiciones sobre el constructo problema.

Fuente: elaborado por el autor

Las posturas mostradas en el Cuadro 2 no son divergentes entre sí, sino que tienen puntos de coincidencia. Sin embargo, algunas de ellas, como las de Rizo y Campistrous (1999) y Cabañas (2000) son poco flexibles, ya que, si se ciñe a esas posturas, en un aula de clases difícilmente se estarán resolviendo problemas. Para efectos de este trabajo, el constructo problema se entiende como García-García, Rodríguez y Navarro (2015).

Asimismo, consistente con García-García (2014a) se habla de resolución de problemas en detrimento de solución de problemas. Esto es así, dado que el primero incluye todas las acciones intencionales que lleva a cabo el estudiante para encontrar la respuesta a la situación que se le plantea, mientras que el segundo se refiere sólo al resultado final, donde poco importa el cómo se procede para llegar a este.

Por otra parte, se asume que los problemas algebraicos son aquellos que requieren un 
modelo algebraico para ser resueltos (desde el punto de vista del profesor o del investigador) o que parece ser la forma más óptima de ser resueltos, por ejemplo, utilizar una ecuación lineal que implica el uso de expresiones algebraicas. Sin embargo, esto no impide que los estudiantes utilicen vías aritméticas para encontrar la solución.

\section{Método de Investigación}

La presente investigación es descriptiva porque busca desarrollar una descripción del fenómeno estudiado a partir de sus características (HERNÁNDEZ; FERNÁNDEZ; BAPTISTA, 2010) y emplea al estudio de casos como método de investigación (KOTHARI, 2004). Este tipo de método implica, según Kothari, una observación cuidadosa y completa de una unidad social, sea una persona, una familia, una institución, un grupo cultural o incluso toda la comunidad.

Para el desarrollo de la investigación se siguió el siguiente esquema metodológico: (1) selección de los casos de estudio, (2) diseño del instrumento para la colecta de datos, (3) aplicación del instrumento, (4) realización de entrevistas para profundizar en las acciones desarrolladas por los estudiantes y, (5) analizar y caracterizar las estrategias encontradas. Estos puntos serán descritos enseguida.

\subsection{Contexto de la investigación y selección de los casos de estudio}

El estudio se desarrolló con un grupo de estudiantes de la UIEG, cuya sede se encuentra en la Región Montaña del estado de Guerrero, México. Esta Universidad atiende a estudiantes que hablan: Tu'un Savi (mixteco), Nāhuatlahtōlli (náhuatl), Me'phaa (tlapaneco), Nomndaa (amuzgo) y el español como lengua materna quienes conviven en un ambiente de respeto, tolerancia, diálogo y solidaridad.

Por esta razón, se asume que la investigación se desarrolló en un contexto intercultural $^{1}$. En la UIEG, los estudiantes se comunican utilizando su lengua materna; además, existen talleres que fomentan el aprendizaje de los diversos idiomas de los estudiantes. La mayor parte de la planta docente también habla una lengua originaria.

La mayoría de los alumnos que llegan a la UIEG no fueron aceptados o tienen un

\footnotetext{
1 Se entiende como una relación entre diversas culturas basada en el respeto y desde planos de igualdad (SCHMELKES, 2013).
} 
promedio por debajo del solicitado en escuelas de nivel superior convencionales. Entre los motivos por el cual eligen esta universidad, destaca en que no se aplica examen de admisión y en su lugar, se realiza una semana de ambientación en la que los futuros estudiantes conocen las licenciaturas que se ofertan, la planta docente, así como las actividades extracurriculares existentes. Los estudiantes que finalmente eligen algunas licenciaturas que oferta la UIEG (Ingeniería Forestal, Desarrollo Sustentable, Lengua y Cultura, Turismo Alternativo, Gestión Local y Gobierno Municipal) son inscritos.

Los estudiantes llegan a la UIEG con un nivel de comprensión distinto en Matemáticas. En particular, los que optan por la licenciatura de Ingeniería Forestal cursan Álgebra, Geometría y Trigonometría y, Cálculo Diferencial e Integral en los semestres 1, 2 y 3 , respectivamente. Posterior a ello, toman cursos propios de su formación profesional donde las Matemáticas son aplicadas, por ejemplo, Dendrometría. El estudio se hizo con estudiantes de primer semestre que eligieron Ingeniería Forestal como opción universitaria, es decir, aquellos que cursaban Álgebra.

En el momento en que se hizo el presente estudio, Ingeniería Forestal tenía una matrícula de siete estudiantes - normalmente esta licenciatura tiene una matrícula baja -; sin embargo, para efectos del presente se eligió a cinco casos (E1, E2, E3, E4, E5). Esta elección obedeció a que eran los estudiantes más constantes. De estos, E2 y E4 hablaban Tu'un Savi (mixteco), E3 y E5 Me'phaa (Tlapaneco) y E1 solo español; sin embargo, la variante era distinta en aquellos que hablaban una lengua originaria. Los participantes respondieron los instrumentos suministrados como parte de su clase de Álgebra. El investigador jugó un doble papel al fungir también como profesor titular.

\subsection{Diseño del instrumento}

Para la colecta de datos se hizo uso de cuestionarios escritos (que plantearon problemas algebraicos) en la lengua oficial (español) que corresponde con la instrucción formal en la UIEG y de entrevistas individuales audio grabadas.

Para elegir los problemas se revisó la literatura que identifica estrategias y que plantea problemas algebraicos, la restricción fue que fueran resolubles utilizando a la ecuación lineal como modelo algebraico y que su nivel de dificultad fuera el menor posible. Esta revisión llevó a adaptar ocho problemas planteados en Cabañas (2000). Sin embargo, después de una prueba piloto con estudiantes de la misma institución, se optó por reducir el instrumento a cinco problemas (Cuadro 3). 


\begin{tabular}{|c|c|}
\hline Número & Problema \\
\hline 1 & $\begin{array}{l}\text { Juan compró cuatro pares de calcetines y dos camisas por } \$ 64 \text {. Sergio compró una } \\
\text { camisa y un par de calcetines por } \$ 24 \text {. ¿Cuánto cuesta un par de calcetines? }\end{array}$ \\
\hline 2 & $\begin{array}{l}\text { Un católico devoto le pidió a la Virgen María que si le duplicaba el dinero que } \\
\text { llevada le daría } \$ 100.00 \text {, el dinero se duplicó y le dio los } \$ 100 \text {; luego se acercó a la } \\
\text { Virgen Juquila y le vuelve a decir lo mismo, de nuevo se duplica el dinero y él le da } \\
\text { los } \$ 100 ; \text { ya para salir, se acerca a la Virgen de la Fátima y le pide lo mismo, se } \\
\text { duplica otra vez el dinero y él le da los } \$ 100 \text {, pero el católico se queda sin dinero. } \\
\text { ¿Qué cantidad de dinero llevaba el católico? }\end{array}$ \\
\hline 3 & $\begin{array}{l}\text { Los hermanos Ana y Pablo recorrieron } 5838 \text { metros en bicicleta para llegar a la } \\
\text { milpa de su papá. Si los dos viajaron en la misma bicicleta y Ana manejó la mitad del } \\
\text { trayecto conducido por Pablo. ¿Cuántos metros les tocó manejar a cada uno? }\end{array}$ \\
\hline 4 & $\begin{array}{l}\text { Cuanto nació Pedro, Juan tenía } 20 \text { años. Ahora Pedro tiene } 3 / 4 \text { de la edad de Juan. } \\
\text { ¿Cuántos años tiene cada uno? }\end{array}$ \\
\hline 5 & $\begin{array}{l}\text { Cinco amigos se reparten pizzas en partes iguales, pero finalizada la repartición llega } \\
\text { un sexto amigo. ¿Qué cantidad de su pizza debe darle cada uno de los cinco amigos } \\
\text { para que los seis les toque la misma cantidad de pizza? }\end{array}$ \\
\hline
\end{tabular}

Cuadro 3 - Problemas algebraicos planteados

Fuente: problemas adaptados de Cabañas (2000)

Los problemas planteados en el Cuadro 3, además de ser resolubles utilizando una ecuación lineal, también admiten el uso de diversas estrategias. Esto se hizo al propósito para que los estudiantes mostraran las diversas formas en que podían hallar la solución.

\subsection{Aplicación del cuestionario y realización de entrevistas}

El cuestionario se aplicó en una sesión con una duración aproximada de dos horas. Esto se hizo para que los estudiantes resolvieran cada problema utilizando diversas vías posibles y sin tener presión alguna por el tiempo. Explícitamente, se les pidió que si conocían más de un procedimiento lo manifestaran y que justificaran, de manera escrita y con la mayor claridad posible, las acciones desarrolladas.

Por su parte, la entrevista se desarrolló un día posterior a la aplicación del cuestionario y fue individual. En ella se buscó profundizar la justificación de las acciones desarrolladas por cada estudiante, principalmente cuando en el cuestionario no eran claras o no las presentaban. La entrevista tuvo una duración promedio de 20 minutos.

\section{Análisis de resultados}

El análisis de las evidencias escritas y orales obtenidas en los cuestionarios y entrevistas se hizo caso por caso y activad por actividad. Esto permitió identificar siete estrategias reflexivas: descompone un factor numérico y lo expresa como una suma de factores, resuelve el problema mediante un tanteo apropiado, se apoya de hechos conocidos y 
genera relaciones, utiliza un modelo algebraico, realiza un trabajo hacia atrás, realiza un reparto proporcional y realiza un reparto equitativo. Además, se identificó el uso de una estrategia irreflexiva: opera de manera incorrecta con los datos dados en el problema. Enseguida se describen estas estrategias por cada tipo de problema.

\subsection{Estrategias que emergieron en la resolución del problema 1}

Descompone un factor numérico y lo expresa como una suma de factores: consiste en que, a partir de un número presente en el texto del problema, el estudiante lo descompone en factores, posterior a ello, considerando las condiciones dadas en el problema lo expresa en términos de una suma de factores (suma de partes de un todo). E1 utilizó esta estrategia (Figura 1) combinándola con la siguiente estrategia.

- Resuelve el problema mediante un tanteo apropiado: emerge cuando el estudiante resuelve el problema por ensayo y error, pero con una orientación correcta (o apropiada); es decir, utiliza las condiciones dadas en el problema, pero limitado por sus conocimientos, recurre a otro tipo de razonamiento que también le lleva a encontrar la solución mediante una aproximación (Figura 1) en lugar de utilizar un modelo algebraico (en nuestro caso).

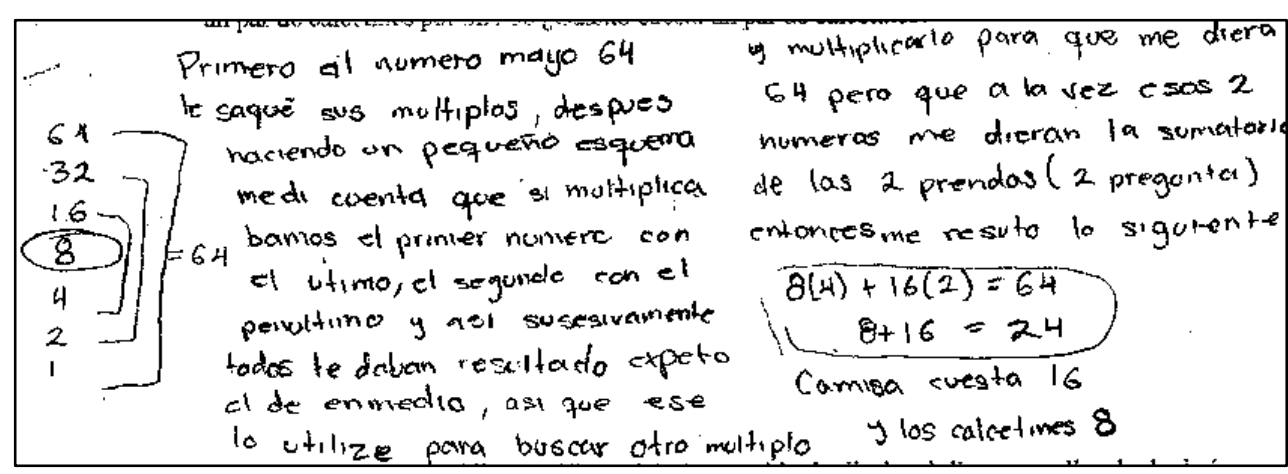

Figura 1 - Resolución hecha por E1

Fuente: Obtenida de las respuestas de los estudiantes

En la Figura 1 se identifica que E1 utiliza la estrategia descompone un factor numérico y lo expresa como una suma de factores cuando ubica el 64 (dato dado en el problema) y lo descompone dividiéndolo entre dos hasta llegar al factor mínimo que es el 1; posterior a ello, expresa ese 64 como una suma de un producto de factores. Para esto último se vale, también, de la estrategia resuelve el problema mediante un tanteo apropiado, es decir, considerando ambas condiciones dadas en el texto del problema y ese 8 que obtuvo al descomponer el 64, deduce el precio que debe tener una camisa y un par de calcetines.

- Se apoya de hechos conocidos y genera relaciones: se manifiesta cuando el estudiante 
utiliza las condiciones dadas en el problema y a partir de ellas genera relaciones entre los datos proporcionados.

Por ejemplo, E2 ubica los datos que ofrece el problema, así como las exigencias que se plantean. A partir de ello, calcula los precios que deben tener una camisa y un par de calcetines. En la entrevista E2 manifestó lo siguiente:

Investigador: ¿qué encuentras primero? ¿El precio del par de calcetines o el precio de la camisa?

E2: el precio de los calcetines.

Investigador: entonces, ¿cómo le haces para encontrar el precio del par de calcetines?

E2: yo sé que una camisa y un par de calcetines cuestan veinticuatro pesos. Entonces, dos pares de calcetines y dos de camisas suman cuarenta y ocho. Como el otro dato me dice que cuatro pares de calcetines y dos camisas cuestan sesenta y cuatro, entonces sólo falta encontrar el precio de los dos pares de calcetines que faltan. El precio de los dos pares lo encuentro restando cuarenta y ocho de sesenta y cuatro, y obtengo dieciséis. Mmm... si dieciséis cuestan dos pares entonces, cada par cuesta ocho pesos.

Investigador: bien y ¿cómo encuentras el precio de una camisa?

E2: como una camisa y un par de calcetines cuestan veinticuatro y, el precio de un par de calcetines cuesta ocho, entonces a veinticuatro le resto ocho y resulta dieciséis. Este resultado es el precio de una camisa.

(Diálogo entre el investigador y el alumno, 2014).

A partir de las explicaciones de E2, se infiere que se apoya del hecho conocido de que una camisa y un par de calcetines cuestan veinticuatro pesos, a partir de ello genera las relaciones pertinentes para encontrar la solución del problema propuesto. En la justificación de E2 se aprecia que su estrategia es reflexiva, porque pasa por un proceso de análisis del problema previamente para deducir las relaciones que establece y luego utiliza.

- Opera de manera incorrecta con los datos dados en el problema: esta estrategia emerge cuando los estudiantes, a partir de los datos que ofrece el problema, realizan al menos una operación básica (suma, resta, multiplicación y división), pero sin un proceso previo de análisis de la situación propuesta.

Por ejemplo, E4 supone inicialmente que el precio de la camisa es \$20. Bajo ese supuesto, y dado que el primer dato que se ofrece en el problema es: Juan compró cuatro pares de calcetines y dos de camisas por $\$ 64$, entonces duplica el 20, cuyo resultado resta de 64, obteniendo 24 por respuesta. Asimismo, considerando que Juan compró 4 pares de calcetines, divide 24 entre 4, obteniendo 6 por resultado, el cual ofrece como solución al precio de calcetines. Por otra parte, puesto que Sergio sólo compro una camisa y un par de calcetines y como E4 asumió que la camisa cuesta 20, entonces resta 20 al 24 (que obtuvo de la resta de 64 menos 40), su respuesta es 4. Finalmente, E4 indica que para Juan el costo de los calcetines fue de $\$ 6.00$ pesos, mientras que para Sergio fue de $\$ 4.00$.

- Utiliza un modelo algebraico (sistema de ecuaciones): consiste en que el estudiante, 
partiendo de los datos que ofrece el problema, utiliza un modelo algebraico para encontrar la solución.

En particular, E5 establece un sistema de ecuaciones lineales con dos incógnitas, aunque errores de tipo algorítmico le impidieron llegar a la solución correcta.

\subsection{Estrategias que emergieron en la resolución del problema 2}

Este problema planteaba una situación imaginaria, pero cuya solución (matemática) podía ser encontrada utilizando una ecuación lineal. Traduciendo al lenguaje algebraico, cada enunciado del problema se forma la ecuación $2[2(2 x-100)-100]-100=0$. Sin embargo, por la complejidad de este modelo algebraico ninguno de los cinco casos utilizó esta vía para llegar a la solución, en cambio, se detectó el uso de las siguientes dos estrategias.

- Realiza un trabajo hacia atrás: esta reside en que el estudiante partiendo de la última condición que se ofrece en el problema realiza un razonamiento inverso, es decir, sigue linealmente las condiciones y operaciones que va indicando el problema, pero en sentido inverso. Considera que su problema ha sido resuelto cuando llega al primer dato o condición que ofrece el problema. Posterior a ello, verifica que su solución cumpla las condiciones dadas. Un ejemplo claro del uso de esta estrategia se percibe en la Figura 2.

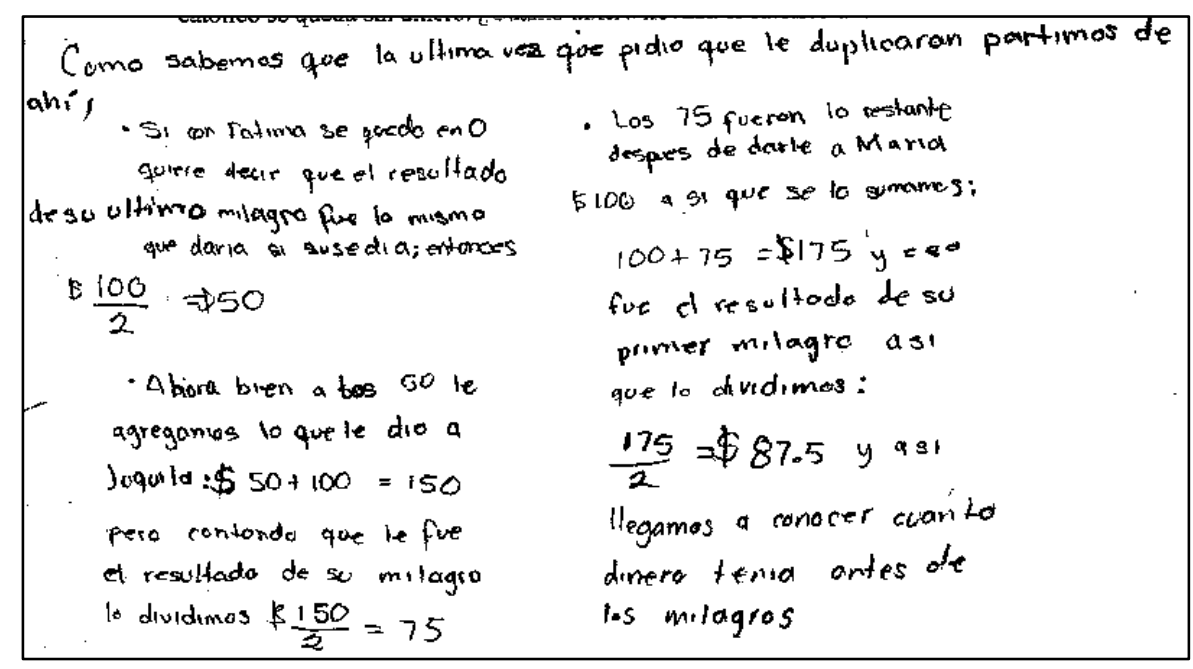

Figura 2 - Resolución de E1 utilizando la estrategia trabajo hacia atrás Fuente: Obtenida de las respuestas de los estudiantes

En la Figura 2, se observa que E1 identifica los datos que ofrece el problema y las restricciones (condiciones) que plantea la situación. E1 parte de la exigencia final y va retrocediendo hasta llegar a la inicial; esto le permite resolver el problema planteado.

- Resuelve el problema mediante un tanteo apropiado: esta estrategia ya fue descrita. 
Es utilizada por todos a excepción de E1 que utilizó la estrategia anterior. Por ejemplo,

E2 durante la entrevista manifestó lo siguiente.

Investigador: ¿Qué hiciste para resolver el problema?

E2: pues busqué el número.

Investigador: pero ¿cómo lo buscaste?

E2: bueno, primero elegí cincuenta, lo duplico y me da cien y le quito cien, entonces me da cero ino me conviene! Ahora, si elijo cien, multiplicado por dos tenemos doscientos, le quito cien me queda cien. Ahora lo duplico otra vez y me da doscientos, le resto otra vez cien, y otra vez me sobra cien. Y así, me queda cien, cien, cien cada vez que duplico y hago la resta, entonces nunca me da cero.

Investigador: en otras palabras, se podría decir que...

E2: mmm... yo pensé en un número que cumpliera lo que dice [el problema]. Empecé con cincuenta, luego con sesenta, setenta, ochenta y ya casi me iba dando. Entonces probé con 90 , ¡se pasaba! Y así me fui acercando.

(Diálogo entre el investigador y el alumno, 2014).

\subsection{Estrategias que emergieron en la resolución del problema 3}

En la resolución de este problema aparecieron las siguientes estrategias.

- Utiliza un modelo algebraico (ecuación lineal): esta estrategia ya fue descrita.

Sin embargo, a diferencia del problema 1, aquí los estudiantes que la utilizan emplean una ecuación lineal como modelo algebraico. Por ejemplo, E1 representa linealmente los datos, al colocar Ana + Pablo $=5838 \mathrm{~m}$ y con base a las exigencias que plantea el problema, recurre a plantear la ecuación $1 / 2+2 / 2 P=5838$. Una vez teniendo lo anterior realiza los cálculos necesarios para ofrecer la respuesta al problema.

- Realiza un reparto proporcional: esta estrategia consiste en que el estudiante, partiendo de las condiciones que plantea el problema, es decir, de un total de 5838 metros recorrido por dos personas, distribuye esa cantidad en partes proporcionales (considerando la condición dada). En otras palabras, divide una magnitud total de manera proporcional, según plantea el problema.

Para ello, E3 se vale de la división y la multiplicación (Figura 3).

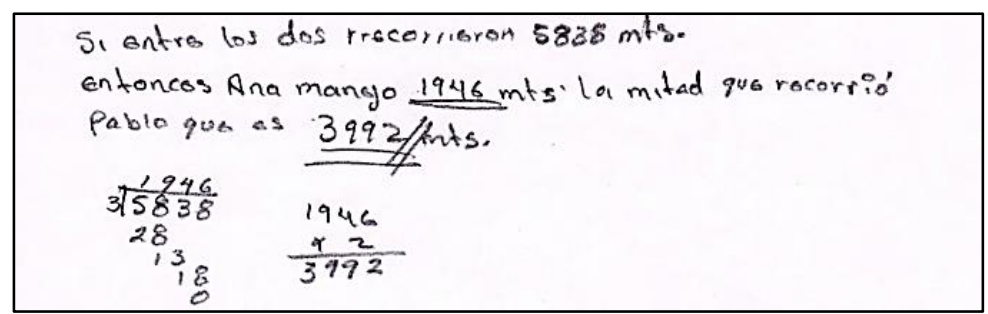

Figura 3 - Procedimiento utilizado por E3 para resolver el problema 3 Fuente: Obtenida de las respuestas de los estudiantes

Se observa, en la Figura 3, que E3 divide 5838 entre 3; el resultado obtenido es la distancia que asume que recorre Ana, mientras que duplica la cantidad para Pablo. 
Por su parte, E4 y E5 operan de manera incorrecta con los datos dados en el problema. E4 toma como referente la cantidad total que recorren en metros Ana y Pablo, es decir, 5838. Esta cantidad la divide entre dos y el resultado lo vuelve a dividir entre dos cuyo resultado asume que es el que recorre Ana. E5 realiza un cálculo análogo.

\subsection{Estrategias que emergieron en la resolución del problema 4}

Todos los estudiantes tuvieron dificultades para resolver este problema. Los cinco operan de manera incorrecta con los datos dados en el problema. Por ejemplo, E1 parte del supuesto de que Pedro tiene 0 años y Juan 20. A partir de ello, divide 20 entre 4, obteniendo 5, el cual multiplica por 3 y afirma que el resultado es la edad de Pedro. Para encontrar la edad de Juan solo suma 20 a 15 que resultó de multiplicar $3\left(\frac{20}{4}\right)$. Es evidente que hizo falta un análisis del problema, puesto que la solución que ofrece el estudiante no cumple las condiciones planteadas en el texto del problema. E2 realiza un procedimiento análogo a E1.

Los restantes (E3, E4 y E5) también parten del supuesto de que Juan tiene 20 años. A partir de ese dato empiezan a operar. E4 divide 20 entre 3 e indica que el resultado es la edad de Pedro. Por su parte E3, suma el 20 con 3/4 para saber la edad de Pedro y para encontrar la de Juan, sólo suma su resultado con 20. En la producción escrita de E3 se identificó que presenta dificultades para operar con números fraccionarios, en particular, para la suma. Por su parte, E5 realiza una resta: $20-3 / 4$. Sin embargo, también presenta dificultades para realizar esta operación, puesto que escribe 17/6 como resultado. El 17 lo obtiene haciendo la resta 20 - 3; mientras que el 6 lo obtiene duplicando el valor del numerador de la fracción 3/4.

\subsection{Estrategias que emergieron en la resolución del problema 5}

Este problema plantea como reto un modelo algebraico cuya ecuación involucra el uso de números fraccionarios. Esa complejidad provocó que E3, E4 y E5 no pudieran resolverla. Mientras que E1 y E2 recurren al uso de la siguiente estrategia:

- Realiza un reparto equitativo: como su nombre lo indica, esta estrategia reside en repartir una cantidad en partes iguales, pero usando el sentido de justica; esto viene planteado como una exigencia en el texto del problema.

En el problema 5 se plantea dividir inicialmente 4 pizzas entre 5 amigos y, posteriormente, a partir de esa división volver a realizar un reparto, pero ahora entre 6 amigos. 
El estudiante E1 toma como referente la cantidad inicial de pizzas (es decir, 4); posterior a ello, realiza lo siguiente:

- Divide esta cantidad entre los primeros 5 amigos. A cada uno le toca $\frac{4}{5}$ de pizzas.

- Luego, al aumentar la cantidad de personas entre las que se deben repartir las pizzas, retoma la fracción anterior y las divide entre seis, resultando $\frac{2}{15}$ (Figura 4).

- Considerando ese resultado (es decir, 2/15) que es la cantidad de pizza que debe darle cada uno de los 5 amigos al sexto que se integra al grupo, multiplica por 5 esa fracción para saber qué cantidad de pizza le corresponde a cada uno de los seis amigos. Su resultado indica que les corresponde $2 / 3$ de las pizzas.

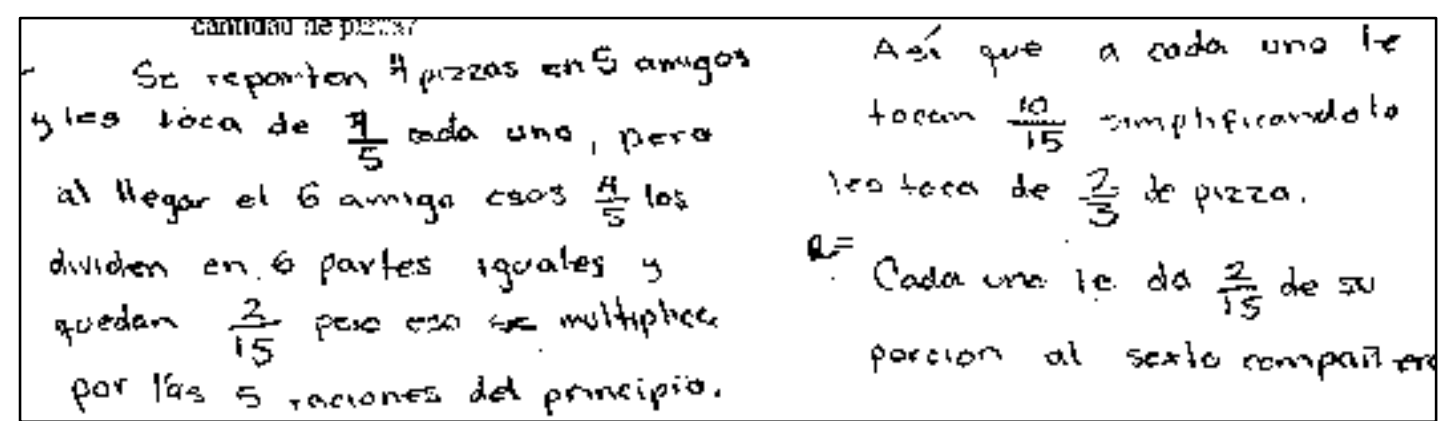

Figura 4 - Reparto equitativo hecha por E1

Fuente: Obtenida de las respuestas de los estudiantes

Lo anterior se refuerza con las respuestas que ofreció E1 durante la entrevista.

Entrevistador: a ver, cuéntame ¿cómo resolviste el problema?

E1: (lee el problema) primero, se reparten cuatro pizzas entre las cinco personas. Bueno, para que a los seis les toque la misma cantidad, primero se reparten las cuatro pizzas entre las cinco personas, les toca, pues, de cuatro quintos a cada uno. Cuando llega el otro, lo que pasa es que, esos cuatro quintos los dividen en seis partes. Una porción que es de cuatro quintos la divido en seis partes y me quedó esto (señala el 2/15 que obtuvo como resultado). Pero eso lo multiplico por las cinco raciones porque los cinco van a darle esa partecita. Así les queda a todos... bueno, simplificando todo, les queda dos tercios de pizza.

Entrevistador: bueno, ¿por qué se te ocurrió escribir cuatro quintos, es decir, dejarlo indicado como una fracción?

E1: porque son cuatro pizzas entre cinco y como no le quise poner valores, lo dejé señalado.

Entrevistador: y luego, ¿por qué divides esa porción entre el total de personas, o sea, entre seis?

E1: porque, llega... mmm... supongamos que cada quien tiene su porción y llega el otro, bueno, a mí se me hizo más fácil que cada quien dividiera su porción en seis y que cada uno se quitara un cachito; todos juntaron esa porción y se la dieron (al que llegó) porque ya iba ser equivalente.

(Diálogo entre el investigador y el alumno, 2014).

Por su parte, E2 también realiza un reparto equitativo. Este opera de manera más directa, es decir, divide las 4 pizzas entre los 5 amigos y, por separado, divide las 4 entre los 6 amigos; finalmente, realiza una resta para saber qué porción le corresponde darle cada uno de los 5 amigos al que recién se integra al grupo. A diferencia de E1, E2 indica que a cada amigo 
le tocaba inicialmente 0.8 partes de la pizza; pero al final, cada amigo debe convidar $0.1 \overline{3}$ de su porción de pizza al sexto. De esta manera, E2 prefiere el uso de números decimales para expresar sus resultados. El procedimiento desarrollado por E2 es el más próximo al modelo algebraico que resuelve problema; parece ser que utilizó este modelo de manera implícita.

\subsection{El contexto intercultural, la lengua materna y la resolución de problemas}

Los resultados permiten plantear algunas reflexiones. La interculturalidad como una relación entre diversas culturas en un ambiente de respeto es fundamental para motivar a los estudiantes a discutir y reflexionar sobre las estrategias para resolver problemas matemáticos (como se identificó posteriormente, en la etapa de instrucción formal). Sin embargo, por la forma de aplicación del cuestionario en esta investigación (etapa previa a la instrucción formal), los estudiantes trabajaron de forma individual, por lo que las estrategias que emergieron fueron personales. Estas tienen dos orígenes: por la enseñanza formal recibida en niveles de educación previos al superior y como una construcción personal del estudiante a partir de su experiencia en contextos escolares y extraescolares.

Como señala García-García (2014a, p. 67), algunos estudiantes "han logrado incorporarse a la práctica castellanizadora de los docentes, quienes los introducen a la cultura dominante”. Esto es, en niveles básicos (preescolar, primaria, secundaria y bachillerato) han sido instruidos en la lengua oficial y bajo contextos - reflejado en los libros de texto, en planes y programas de estudio, en planeaciones didácticas etc.- ajenos a su cultura. Como consecuencia, han logrado integrarse a esa cultura aprendiendo una segunda lengua, resolviendo problemas que no tienen sentido en su contexto cultural, pero también aprendiendo estrategias para resolver problemas que sus profesores les enseñaron. Esto justifica que los casos de estudio emplearan estrategias similares al resolver ciertos problemas, por ejemplo, resuelve el problema mediante un tanteo apropiado y opera de manera incorrecta con los datos dados en el problema.

Un hecho importante a resaltar a partir de las respuestas de E2 es que él identifica, claramente, la distinción entre resolver un problema que tiene sentido matemáticamente, pero que es imaginario o irreal. Por ejemplo, en el problema 2 que planteaba la existencia de un milagro para duplicar el dinero de un creyente, el estudiante afirma en la entrevista que es posible que éste no llevara dinero alguno (cuando se le plantea en el problema ¿Qué cantidad de dinero llevaba el creyente?) porque no suceden esas cosas haciendo referencia al milagro.

Este estudiante, por ejemplo, pese a pertenecer a la cultura Ñu Savi que profesa 
diversas costumbres y tradiciones religiosas, niega lo que en su cultura representaría la fe, es decir, la existencia de milagros. Pero, influenciado por el contexto escolar, resuelve el problema porque para él sí tiene sentido en el contexto escolar.

Este tipo de problemas - irreales para el estudiante -, así como algunos similares, representan un claro ejemplo de situaciones que se resuelven normalmente en una clase de matemáticas, pero que, si no exploramos previamente las creencias de los estudiantes asociados a estos, podemos ignorar que para ellos no tiene sentido resolverlos porque están desconectados de $s u$ realidad y de su contexto. Esto, creemos que desarrolla en ellos la creencia de que las matemáticas significa operar con números (sin importar si tiene sentido o no el problema de donde se extraen). Esto último justificaría el alto porcentaje de estudiantes que resuelve el problema que se presentó en la sección de introducción.

Las dificultades que presentaron algunos casos de estudio en la presente investigación se acentúan más por cuestiones lingüísticas (por ejemplo, E3, E4 y E5). Esto provoca una mala comprensión del problema planteado, dificultad para extraer los datos que ofrece o bien para entender las condiciones que establece el mismo. Esto los lleva a operar de manera incorrecta con los datos que ofrecen los problemas. La cuestión lingüística también afecta en el momento de traducir el texto del problema al lenguaje algebraico. Como consecuencia, apareció con menor frecuencia el uso de la estrategia que emplea un modelo algebraico.

En relación con la cuestión lingüística, se reconoce que una limitante del instrumento utilizado fue que no se pudo plantear en la lengua materna de los casos de estudio ni fue posible traducirle el texto del problema durante la aplicación porque el investigador sólo dominaba la variante del Tu'un Savi de E4. Es posible que un mayor control sobre esta variable pudo permitir mejores resultados. Sin embargo, en la instrucción formal se superó este obstáculo porque los estudiantes se comunicaban en su lengua materna para explicar a sus pares cuando estos no comprendían los conceptos matemáticos o las estrategias trabajadas en el aula.

\section{Discusión y conclusión}

Los resultados indican que los estudiantes hicieron uso de siete estrategias reflexivas y una irreflexiva (Tabla 1). Esta última tipología emerge con mayor frecuencia en los problemas 4 y 5 , además, el quinto problema no fue resuelto por tres estudiantes (Tabla 1). 
Tabla 1 - Tipología de estrategias identificadas en los casos de estudio

\begin{tabular}{|c|c|c|c|c|c|c|c|}
\hline & & \multicolumn{5}{|c|}{ Problemas } & \multirow{2}{*}{$f$} \\
\hline \multicolumn{2}{|r|}{ Estrategias } & P1 & $\mathbf{P 2}$ & P3 & P4 & P5 & \\
\hline \multirow{7}{*}{$\frac{\pi}{\sum_{x}^{2}}$} & $\begin{array}{l}\text { Descompone un factor numérico y lo } \\
\text { expresa como una suma de factores. }\end{array}$ & E1 & & & & & 1 \\
\hline & $\begin{array}{l}\text { Resuelve el problema mediante un tanteo } \\
\text { apropiado. }\end{array}$ & E1 & $\begin{array}{l}\mathrm{E} 2, \mathrm{E} 3, \\
\mathrm{E} 4, \mathrm{E} 5\end{array}$ & & & & 5 \\
\hline & $\begin{array}{l}\text { Se apoya de hechos conocidos y genera } \\
\text { relaciones. }\end{array}$ & E2 & & & & & 1 \\
\hline & Utiliza un modelo algebraico. & E5 & & $\mathrm{E} 1, \mathrm{E} 2$ & & & 3 \\
\hline & Realiza un trabajo hacia atrás. & & E1 & & & & 1 \\
\hline & Realiza un reparto proporcional. & & & E3 & & & 1 \\
\hline & Realiza un reparto equitativo. & & & & & $\begin{array}{l}\text { E1, } \\
\text { E2 }\end{array}$ & 2 \\
\hline \multirow[t]{2}{*}{ 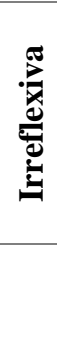 } & $\begin{array}{l}\text { Opera de manera incorrecta con los datos } \\
\text { dados en el problema (una o más operación } \\
\text { básica: suma, resta, multiplicación o } \\
\text { división). }\end{array}$ & $\begin{array}{l}\text { E4, } \\
\text { E3 }\end{array}$ & & $\mathrm{E} 4, \mathrm{E} 5$ & $\begin{array}{l}\text { E1, E2, } \\
\text { E3, E4, } \\
\quad \text { E5 }\end{array}$ & & 9 \\
\hline & No resuelve & & & & & $\begin{array}{l}\text { E3, } \\
\text { E4, } \\
\text { E5 }\end{array}$ & 3 \\
\hline
\end{tabular}

Significado de la simbología: $\mathrm{E}_{i}=$ estudiante caso, $\mathrm{P}_{i}=$ número del problema, $f=$ frecuencia

Fuente: elaboración propia

La Tabla 1, también indica que sólo E1 combina dos estrategias para resolver el primer problema. Estos resultados nos invitan a reflexionar sobre la justificación que ofrece el estudiante al procedimiento algorítmico que realiza. Algunas veces, puede estar utilizando una estrategia irreflexiva, pero llegar a una solución correcta. Esto desarrollaría en él creencias matemáticas que, para conceptos posteriores, le llevarían a desarrollar una concepción alternativa. Por ejemplo, pese a que E3 encontró la solución correcta del primer problema, sus argumentos fueron inconsistentes desde el punto de vista de las matemáticas, por lo que su estrategia fue operar de manera incorrecta con los datos dados en el problema (Tabla 1). Esto nos invita como profesores a indagar más allá de la solución que el estudiante ofrece para ayudarlo a desarrollar un razonamiento matemático apropiado.

De las estrategias presentadas en la Tabla 1, las estrategias descompone un factor numérico y lo expresa como una suma de factores, se apoya de hechos conocidos y genera relaciones y realiza un reparto proporcional son aportes de este trabajo en cuanto estrategias para resolver problemas algebraicos. En cambio, estrategias como realiza un trabajo hacia atrás es utilizada por profesores cuando éstos resuelven problemas aritméticos (OCAMPO, 2000) y resuelve el problema mediante un tanteo apropiado y opera de manera incorrecta con los datos dados en el problema son reportadas en Rizo y Campistrous (1999) cuando exploraron las estrategias que estudiantes mexicanos y cubanos emplean al resolver 
problemas matemáticos.

Asimismo, resuelve el problema mediante un tanteo apropiado y opera de manera incorrecta con los datos dados en el problema son estrategias que también emergen con estudiantes Tee Savi (mixtecos) de primaria cuando resuelven problemas aritméticos (GARCÍA-GARCÍA， 2014a; GARCÍA-GARCÍA; NAVARRO; RODRÍGUEZ， 2014; GARCÍA-GARCÍA; RODRÍGUEZ; NAVARRO, 2015). La primera de estas tiene potencial para conducir al éxito en la resolución de los problemas según Elia, Heuvel-Panhuizen e Kolovou (2009). Los resultados obtenidos en este estudio indican que también es exitosa cuando se resuelven problemas algebraicos, siempre que el estudiante realice un análisis previo del problema planteado.

Por otra parte, a partir de la clasificación hecha por Jiang y Chua (2010), las estrategias que se categorizaron como: realiza un reparto equitativo, resuelve el problema mediante un tanteo apropiado, se apoya de hechos conocidos y genera relaciones y, realiza un reparto proporcional pueden ser consideradas de tipo aritméticas porque emplean sólo el uso de las operaciones básicas con valores numéricos, mientras que la de utiliza un modelo algebraico se puede caracterizar como una estrategia algebraica.

Jiang y Chua (2010) reportan que los estudiantes chinos de sexto grado de nivel primaria utilizaron con mayor frecuencia las estrategias algebraicas para resolver problemas en comparación con los de Singapur. En cambio, en esta investigación se identificó mayor presencia de las estrategias aritméticas al resolver problemas algebraicos.

En Obersteiner, Bernhard y Reiss (2015), un estudio realizado con niños de primaria, indican que, entre otras razones, ignorar la información pertinente planteada en el problema explica el uso de algunas estrategias que son guiadas por la intuición de los niños. Los resultados obtenidos en esta investigación, por su parte, señalan que en los estudiantes de nivel superior sucede algo similar. Cuando ignoran algunas de las condiciones planteadas en los problemas tienden a usar una estrategia irreflexiva para encontrar la solución, en particular, operan de manera incorrecta con los datos presentes en el texto del problema.

Por otra parte, el desempeño de los estudiantes, manifestado a través del uso de las estrategias reflexivas, tuvo que ver en parte con los antecedentes escolares de los mismos y el gusto que manifestaban por las Matemáticas. E1 y E2 demostraron, durante el semestre, una mayor participación en las discusiones matemáticas que fueron propiciadas por el profesor, así como mayores habilidades para resolver tareas matemáticas.

Mientras que E3, E4 y E5 participaron con mayores dificultades, pero con disposición para integrarse a las discusiones grupales. El contexto escolar, así como la relación entre los 
alumnos permitió esta integración para practicar la interculturalidad.

Algunas estrategias identificadas en los casos de estudio (por ejemplo, las de trabajo hacia atrás, realiza un reparto proporcional y resuelve el problema mediante un tanteo apropiado) fueron incorporadas durante las lecciones formales cuando se resolvieron problemas algebraicos. Esto se hizo para motivar la participación de los estudiantes a compartir sus conocimientos y estrategias personales para resolver problemas específicos, pero también para fomentar la discusión sobre la necesidad de aprender estrategias más óptimas para resolver los problemas algebraicos. Asimismo, ayudó a evitar que los alumnos fueran meros receptores de las estrategias sugeridas por el profesor.

Esto favoreció la práctica de la interculturalidad; los alumnos escuchaban a sus compañeros, discutían la propuesta de sus pares en un ambiente de respeto y manifestaban con libertad los conocimientos matemáticos utilizados en sus culturas. Se debe admitir que la baja matrícula permitió que esto se desarrollara con mayor éxito.

En el nivel superior, a partir de los casos de estudio y sin pretender generalizar, podemos plantear que la instrucción escolar ha permeado en las estrategias que los estudiantes han consolidado (como resuelve el problema mediante un tanteo apropiado y utiliza un modelo algebraico). Sus antecedentes escolares (bueno, regular o deficiente desempeño en matemáticas), los conocimientos matemáticos de que disponen, la cuestión linguiística (en algunos), el gusto por las matemáticas y, de alguna manera, el contexto implícito en el problema (como lo reconocen CARRAHER; CARRAHER; SCHLIEMANN, 2007; BLANCO; BLANCO, 2009; GARCÍA-GARCÍA, 2014a, 2014b) también influye en su éxito para resolver problemas matemáticos. Sus dificultades matemáticas están más asociadas a la operatividad con fracciones y con números enteros.

Finalmente, los resultados obtenidos en esta investigación indican que es necesario seguir profundizando en el estudio de las estrategias para comprender el proceder de los estudiantes indígenas en el momento de resolver problemas. Será importante, en estudios posteriores, identificar las matemáticas presentes y utilizadas en las culturas de los estudiantes, como su sistema de numeración (como el estudio de GARCÍA-GARCÍA, 2015) para incorporar esos conocimientos durante el proceso enseñanza-aprendizaje. Estos conocimientos podrían ser aprovechados - incluso en otros contextos escolares con diversidad cultural - en el proceso de enseñanza-aprendizaje de distintos conceptos matemáticos.

\section{Referencias}

BERMEJO, V.; DÍAZ, J. J. The degree of abstraction in solving addition and subtraction problems. 
The Spanish Journal of Psychology, London, v. 10, n. 2, p. 285-293, 2007.

BLANCO, B.; BLANCO, L. J. Contextos y estrategias en la resolución de problemas de primaria. Revista Números, España, v. 71, p. 75-85, 2009.

CABAÑAS, M. G. Los problemas... ¿cómo enseño a resolverlos? 1. ed. México: Grupo Editorial Iberoamericana, 2000. $50 \mathrm{p}$.

CARRAHER, T.; CARRAHER, D.; SCHLIEMANN, A. En la vida diez, en la escuela cero: Los contextos culturales del aprendizaje de las matemáticas. En: CARRAHER, T.; CARRAHER, D.; SCHLIEMANN, A. (Ed.). En la vida Diez, en la escuela cero. México: Siglo XXI Editores, 2007. p. $25-47$.

CHE, M.; WIEGERT, E.; THRELKELD, K. Problem solving strategies of girls and boys in single-sex mathematics classroom. Educational Studies in Mathematics, Netherlands, v. 79, n. 2, p. 311-326, 2012.

DÍAZ-BARRIGA, F.; HERNÁNDEZ, G. Estrategias docentes para un aprendizaje significativo: Una interpretación constructivista. 2. ed. Distrito Federal, México: Editorial Mc Graw Hill, 2010. 465 p.

ECHENIQUE, I. Matemáticas resolución de problemas. 1. ed. Navarra, España: Fondo de publicaciones del gobierno de Navarra, 2006. 162 p.

ELIA, I.; HEUVEL-PANHUIZEN, M.; KOLOVOU, A. Exploring strategy use and strategy flexibility in non-routine problem solving by primary school high achievers in mathematics. ZDM Mathematics Education, Germany, v. 41, p. 605-618, 2009.

FERNÁNDEZ, J. A. Algo sobre resolución de problemas matemáticos en educación primaria. Revista Sigma, España, v. 29, p. 29-42, 2006.

GARCÍA, J. Estrategias en la resolución de problemas aritméticos: el caso de los niños mixtecos. 2012. 129f. Tesis (Maestría en Ciencias Área: Matemática Educativa) - Universidad Autónoma de Guerrero, México, 2012.

GARCÍA, J. La resolución de problemas formales y prácticos: un estudio con niños Tee Savi. Revista números, España, v. 84, p. 25-45, 2013.

GARCÍA-GARCÍA, J. De los problemas prácticos a los formales: una transición necesaria en el aula de clases. En: LESTÓN, P. (Ed.). ACTA LATINOAMERICANA DE MATEMÁTICA EDUCATIVA, 27, 2014, México: Colegio Mexicano de Matemática Educativa A. C. y Comité Latinoamericano de Matemática Educativa A. C., 2014b. p. 181-190.

GARCÍA-GARCÍA, J. El contexto cultural y la resolución de problemas: vistos desde el salón de clases de una comunidad Ñuu Savi. Revista Latinoamericana de Etnomatemática, Colombia, v. 7, n. 1 , p. 50-73. 2014a.

GARCÍA-GARCÍA, J. El sistema de numeración vigesimal: ¿cómo utilizarlo en el aula? Revista Novedades Educativas, Argentina, v. 292, n. 7, 76-79, 2015.

GARCÍA-GARCÍA, J.; NAVARRO, C.; RODRÍGUEZ, F. M. La resolución de problemas en un contexto Nuu Savi: un estudio de casos con niños de sexto grado de primaria. Educación Matemática, México, v. 26, n. 1, 127-152, 2014.

GARCÍA-GARCÍA, J.; RODRÍGUEZ, F. M.; NAVARRO, C. Las estrategias utilizadas por los niños 
Tee Savi en la resolución de problemas aritméticos. Revista Latinoamericana de Investigación en Matemática Educativa, México, v. 18, n. 2, p. 213-244, 2015.

HERNÁNDEZ, R.; FERNÁNDEZ, C.; BAPTISTA, P. Metodología de la investigación. 5.ed. México: Mc Graw Hill, 2010. 613 p.

JIANG, C.; CHUA, B. L. Strategies for solving three fraction-related word problems on speed: a comparative study between Chinese and Singaporean students. International Journal of Science and Mathematics Education, Taiwan, v. 8, p. 73-96, 2010.

KOTHARI, C. R. Research Methodology: Methods and Techniques. 2. ed. Jaipur, India: New Age International Publishers, 2004. 400 p.

MOLINA, M.; AMBROSE, R. El papel del lenguaje en la resolución de problemas verbales aritméticos. Un estudio con niños bilingües. En: MORENO, M. M. et al. (Ed.). Investigación en Educación Matemática XIV. Lleida: Sociedad Española de Investigación en Educación Matemática, 2010. p. 423-434.

OBERSTEINER, A.; BERNHARD, M.; REISS, K. Primary school children's strategies in solving contingency table problems: the role of intuition and inhibition. ZDM Mathematics Education, Germany, v. 47, p. 825-836, 2015.

OCAMPO, M. Caracterización de las estrategias que utilizan los profesores al enseñar a resolver problemas aritméticos: Un estudio de casos. 2000. 105f. Tesis (Maestría en Ciencias Área: Matemática Educativa) - Universidad Autónoma de Guerrero, México, 2000.

ORTIZ, F. Matemática: estrategias de enseñanza y aprendizaje. 1. ed. México: Editorial Pax, 2001, $141 \mathrm{p}$.

RIZO, C.; CAMPISTROUS, L. Estrategias de resolución de problemas en la escuela. Revista Latinoamericana de Investigación en Matemática Educativa, México, v. 2, n. 2-3, p. 31-45, 1999.

SANTOS, L. M. La resolución de problemas matemáticos: fundamentos cognitivos. 1.ed. México: Editorial Trillas, 2010. 254 p.

SCHMELKES, S. Educación para un México intercultural. Revista Electrónica Sinéctica, v. 1, n. 40, enero-junio, p. 1-12, 2013.

SCHOENFELD, A. Mathematical Problem Solving. 1. ed. New York: Academic Press, 1985. 409 p.

VILLALOBOS, X. Resolución de problemas matemáticos: un cambio epistemológico con resultados metodológicos. Revista Iberoamericana sobre Calidad, Eficacia y Cambio en Educación, Iberoamérica, v. 6, n. 3, p. 37-58, 2008.

Submetido em 09 de Fevereiro de 2018. Aprovado em 12 de Junho de 2018. 